\title{
GLAZED OPENINGS' DESIGN AND ITS EFFECT ON ENERGY CONSUMPTION IN SAUDI ARABIA
}

\section{Khalid Alshaibani}

College of Architecture and Planning, King Faisal University, Dammam,

Saudi Arabia

Email:kshaibani@gmail.com

(Received May 25, 2008 Accepted July 28, 2008)

The running cost of residential buildings could be one of the important tools that can support the concept of the Affordable Housing. This is because residential buildings consume almost 50\% of total produced electricity in the kingdom. Several studies has shown that heat gains through external envelope can reach up to $75 \%$ of the total heat loads of the building. Therefore, saving energy in buildings can start from the external envelope and its components such as form, orientation, material and the fenestrations.

This paper aims to investigate the effect of glazing openings on energy consumption in residential buildings in Saudi Arabia.

KEYWORDS: Glazing, thermal insulation, residential buildings, energy consumption in residential buildings.

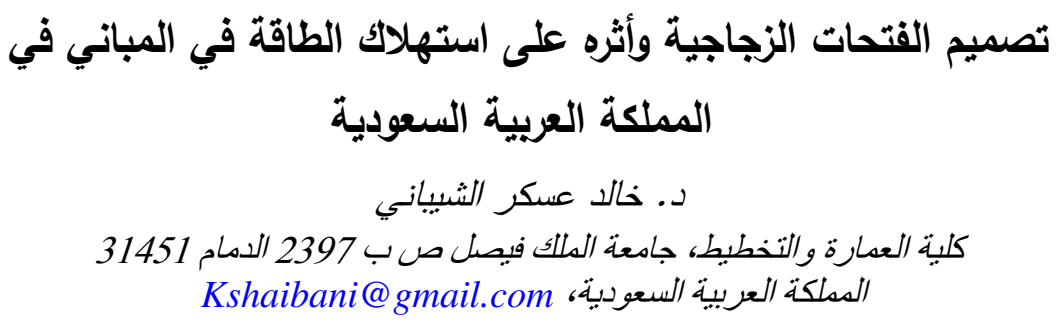

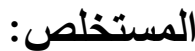

تتشكل التكلفة التشغيلية للمسكن إحدى الآليات التي من المدكن أن تدعم مفهوم المسكن الميسر . فالمباني السكنية تستهلك ما يقارب 50\% من إجمالي من الطاقة الكهربائية المنتجة في المملكة العربية السعودية. وتختلف مساهمات مكونات المبنى في الأحمال الحرارية باختلاف فئات المباني والنشاطات الممارسة، وتثير هذه الدراسات إلى أن مساهمات الغلاف الخارجي تصل إلى أكثر من 50\% من إحسات إجمالي الأحمال

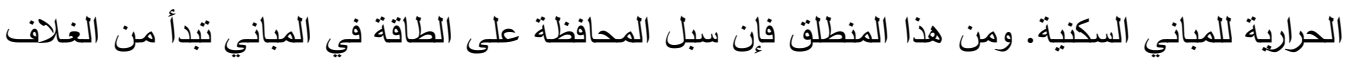
الخارجي ومكوناته الرئيسية والتي تشمل العديد من العناصر كالثكل الهندسي للمبنى واتجاهه والخواص التصال

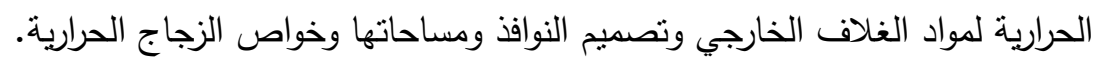

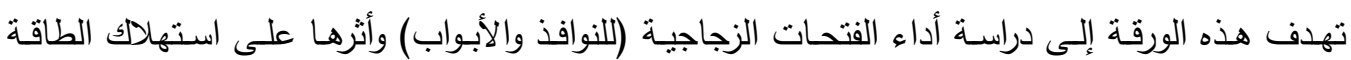
الكهربائية في المباني السكنية في المملكة العربية السعودية. 
الكلمات الدالة: الفتحات الزجاجية، العزل الحراري، المباني السكنية، استهلاك الطاقة في المباني.

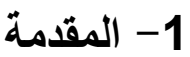

ييرز الطلب المتزايد على الطاقة الكهربائية في المملكة العربية السعودية بصورة واضحة حيث من المتوقع أن بصل إلى 60 جيجا واط بحلول 2023م منطلبا بذللك استثمارات تقدر ب 340 مليار ربال [1]، [2]. ويثكل هذا الرقم من الطاقة ثلاثة أضعاف الطاقة المنتجة في عام 1998. وتمثل هذه الأرقام تحديا كبيرا لثركة الكهرباء والمسئولين عن نوفير الطاقة الكهربائية بالمملكة. ولقد تبنت الثركة السعودية للكهرباء مؤخرا خطة تنتد إلى عام 2015 م تكلف ما يقارب 190 مليار ريال تثمل مشاريع التوليد والنقل والتوزيع [3]. ويوضح شكل 1 التطور في الطاقة الكهربائية المباعة حتى 2006 والتوقعات المستقبلية حتى بـى 2011م بنسبة نمو نقارب الـ 6\% سنويا.

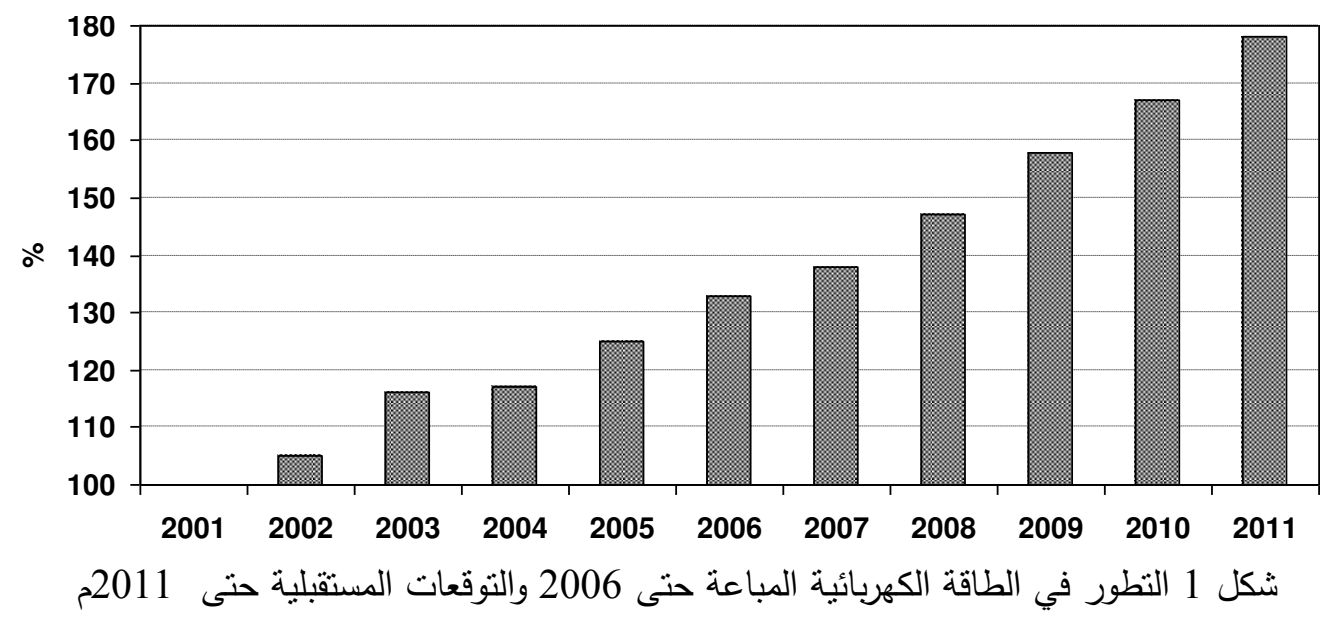

وتتكل المباني المستهلك الأكبر للطاقة الكهربائية في المملكة، حيث تستهلك ما يصل إلى 75\% من الطاقة الكهربائية وتصل حصة المباني السكنية إلى ما يقارب ال52\% من الطاقة الكهربائية المستهلكة بالمملكة (الثكل2).

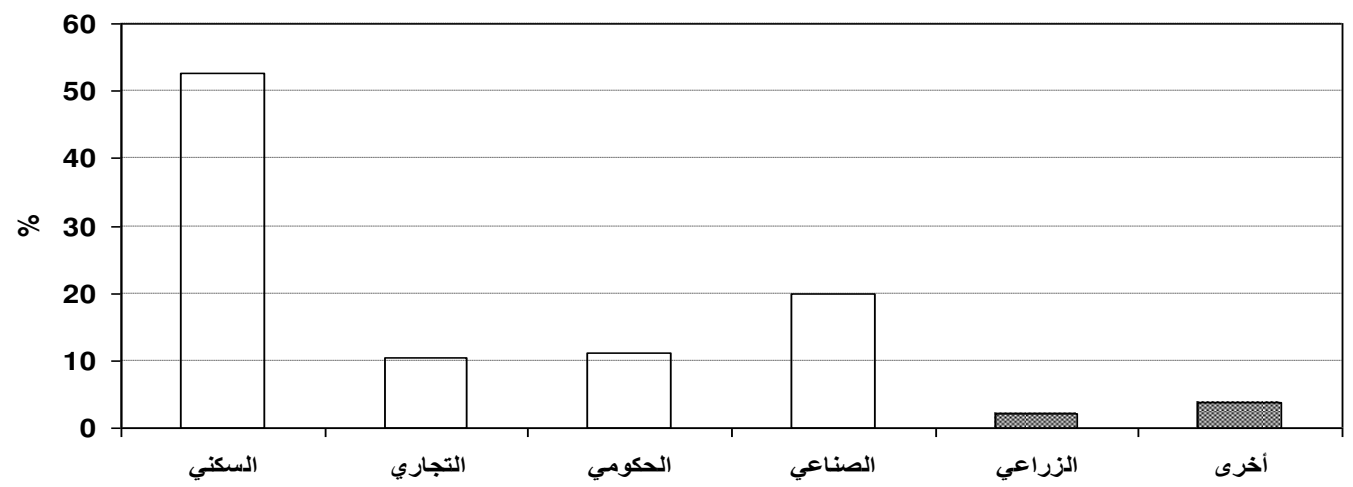

شكل 2 نسب استهلاك الطاقة الكهربائية حسب فئات المشتركين لعام 2006 [3] 
ولفهم أثر تصميم المبنى ومكوناته على استهلاك الكهرباء في المملكة العربية السعودية أجريت العديد

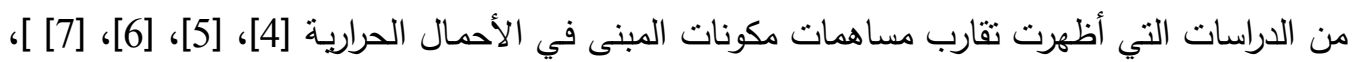

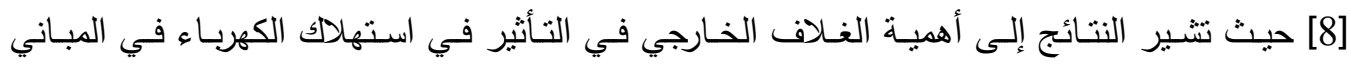
السكنية، حيث يمكن أن يصل أثره إلى أكثر من 50\% (الثنكل

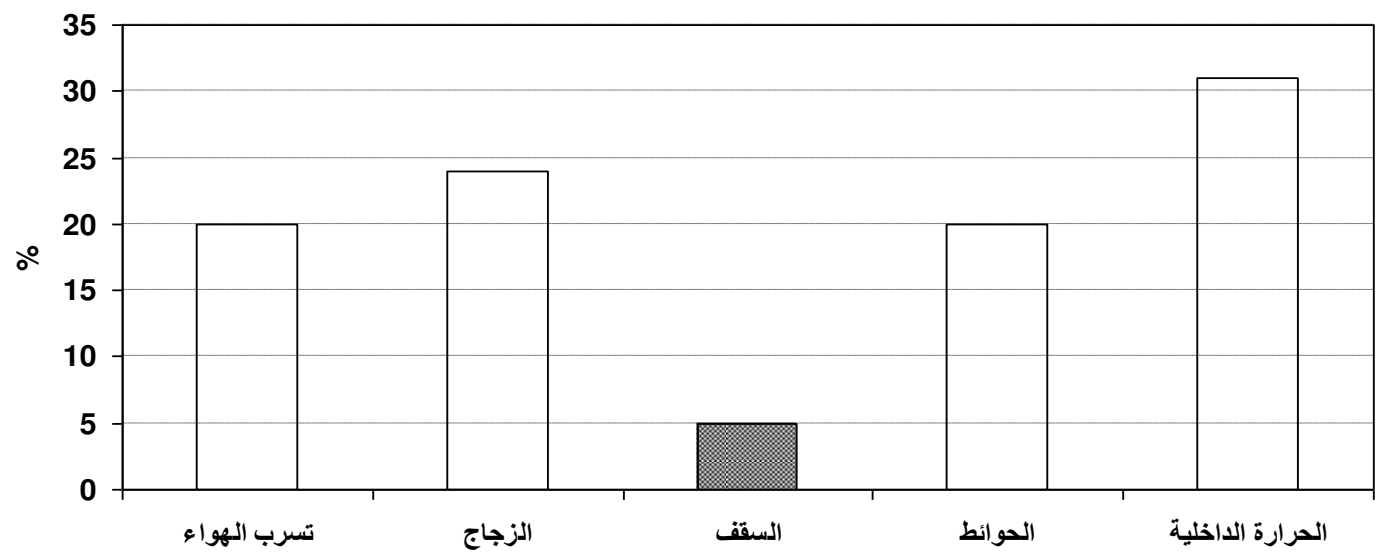

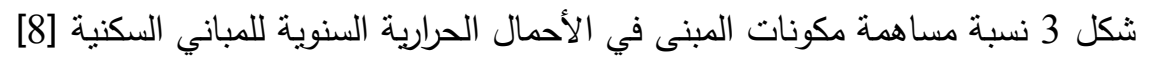

وتركز هذه الدراسة على عنصر أساسي من عناصر الغلاف الخارجي ألا وهو الفتحات الزجاجية وذلك لفهم تأثير تصميم الفتحات الزجاجية على استهلاك الطاقة الكهربائية في المباني السكنية.

\section{2- منهجية الدراسة}

تم خـلال هذه الدراسـة استخدام برنـامج محاكاة أداء الطاقة (Visual Doe-V4.0) في تحليل أداء

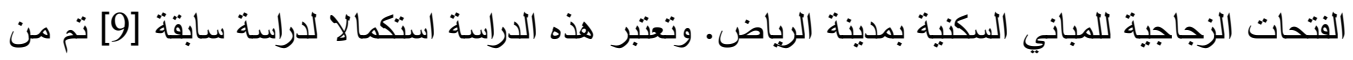

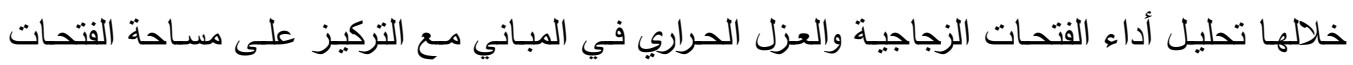

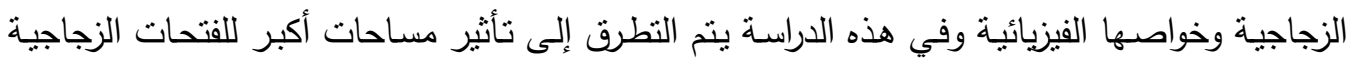

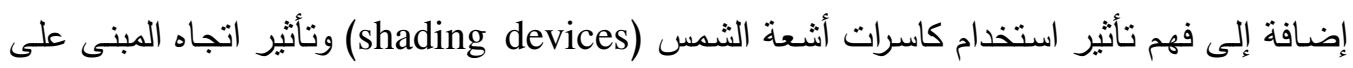
استهلاك الطاقة الكهربائية في المباني السكنية. ويوضح الجدول 1 المعطيات الرئيسية المستخدمة في 
جدول 1 المعطيات الرئيسية المستخدمة في الدراسات التمثيلية

\begin{tabular}{|c|c|c|}
\hline مبنى سكني & نوع المبنى & 1 \\
\hline مدينة الرياض & موقع المبنى & 2 \\
\hline دورين & عدد الأدوار & 3 \\
\hline 225 متر مربع & مساحة الدور & 4 \\
\hline (450 منر مربع & المساحة الإجمالية للأدوار & 5 \\
\hline 3.25 & ارتفاع الدور & 6 \\
\hline 6.5 & ارتفاع المبنى & 7 \\
\hline 2.93 & معامل الموصلية الحرارية للحوائط (U factor) & 8 \\
\hline 2.08 & معامل الموصلية الحرارية للسقف & 9 \\
\hline البوليسترين & نوعية العازل الحراري & 10 \\
\hline 0.0346 . & معامل الموصلية الحرارية للعازل الحراري & 11 \\
\hline 6.2 & معامل الموصلية الحرارية للزجاج & 12 \\
\hline 0.82 & معامل الاكتساب الحراري الثمسي للزجاج (SHGC) & 13 \\
\hline
\end{tabular}

\section{3- الاراسات التحليلية}

\section{3-1 أثر مساحة القتحات الزجاجية}

ركزت الدراسات التحليلية الأولية على أثز مساحة الفتحات الزجاجية على استهلاك الطاقة الكهبائية في المباني السكنية. وتراوحت مساحات الفتحات الزجاجية المستخدمة من 5\% إلى 60\% من مساحة المبنى. وأظهرت هذه الدراسات أهمية مساحة الفتحات الزجاجية في التأثثر على استهلاك الطاقة الكهربائية. فالثكل 4 يوضح مدى أهمية مساحة الفتحات الزجاجية في التأثير على استهلالك الطاقة الكهربائية في المباني السكنية، حيث أن زيادة نسبة مساحة الفتحات الزجاجية بالنسبة لمساحة المبنى من 5\% إلى 50\% من

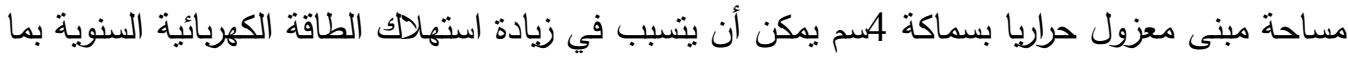
يقارب من 55\% من قيمة الاستهلاك. أما في حالة المبنى غير المعزول حراريا فتؤدي نفس الزيادة في مساحة الفتحات الزجاجية بالنسبة لمساحة المبنى (من 5 \% من إلى 50\%) اللى مئى زيادة الاستهلالك الكهربائي السنوي بما يقارب 30\% من إجمالي الاستهلاك السنوي. ومعنى ذلك أن تأثير زيادة مساحة النوافذ يكون أكبر في المباني المعزولة عنه في المباني غير المعزولة. 


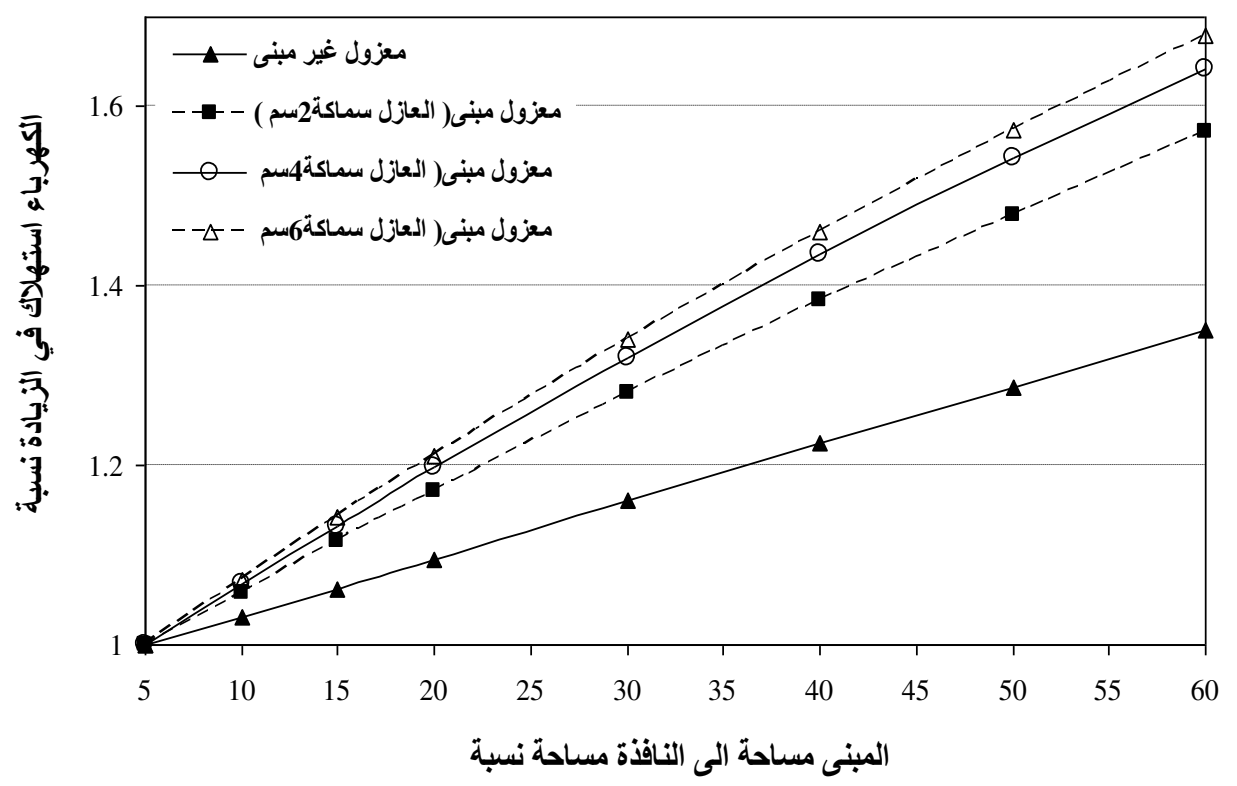

شكل 4 أثر مساحة الفتحات الزجاجية على الاستهلاك الكهربائي السنوي

ويظهر الثكل 5 نتائج أثر الزيادة في مساحة الفتحات الزجاجية على أداء العازل الحراري، حيث يمكن

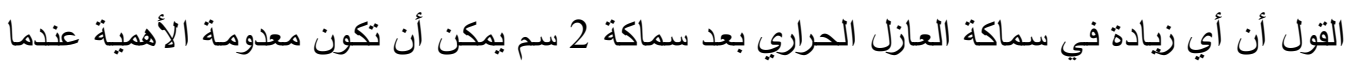

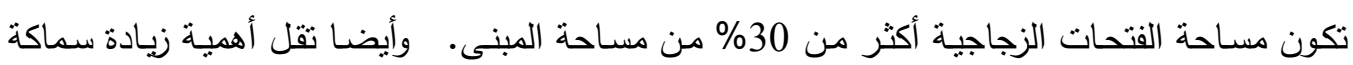

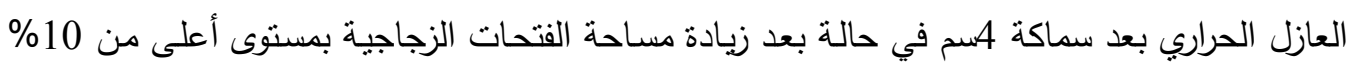

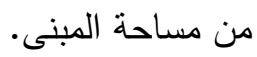
وبمقارنـة الثكلين 4 و 5 يمكن التأكيد على أهمية مسـاحة الفتحات الزجاجية. فعلى سبيل المثنال ومن الثكل 5 يمكن القول أنه بزيادة سماكة العازل الحراري من 2سم إلى 7سم لمبنى سكنى سكني بمساحة 20\%

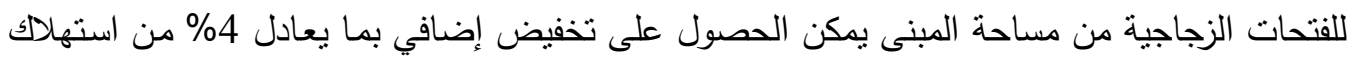

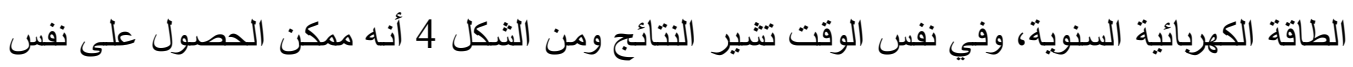
التخفيض في استهلاك الكهرباء السنوي وذلك بالتقليل من مساحة الفتحات الزجاجية من نسبة 20\% من من

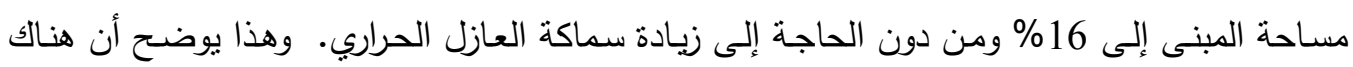
خيارات مختلفة وبتكلفة أقل للتعامل مع استهلاك الطاقة الكهربائية. 


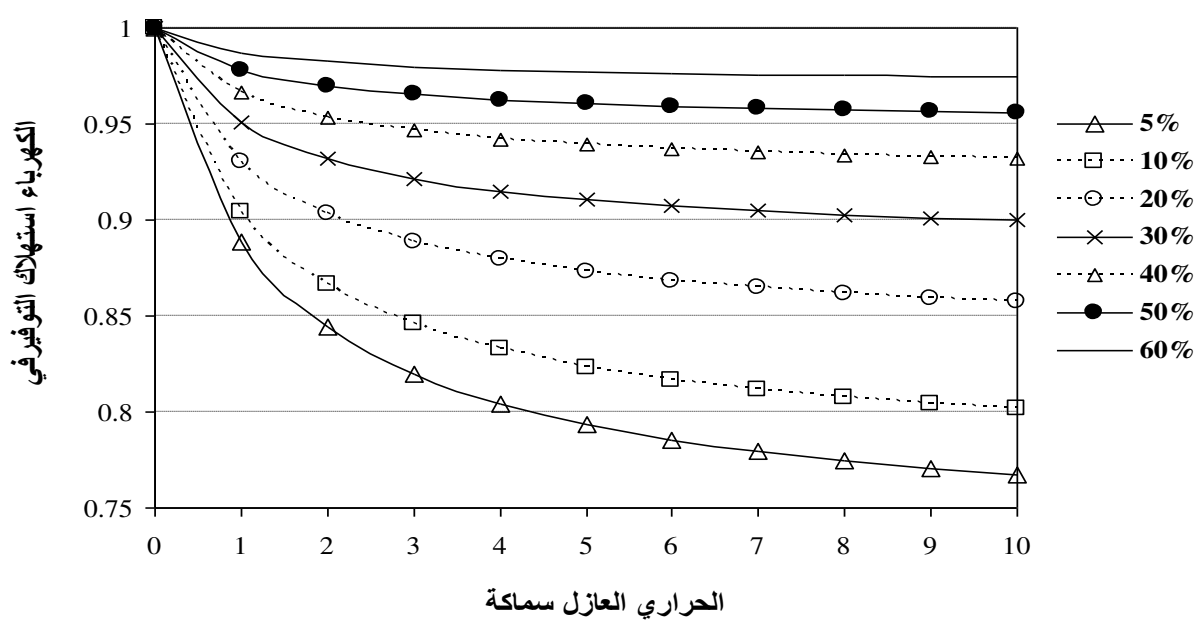

شكل 5 أنز مساحة القتحات الزجاجية على أداء العازل الحراري

\section{3-2 توجيه الفتحات الزجاجية}

تم من خـلال هذه الدراسـة تحليل أثر توجيه الفتحات الزجاجية على استهلالك الطاقة الكهربائية. ففي

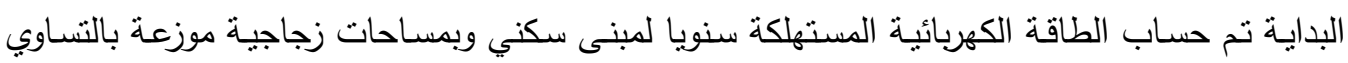
على الواجهات الأربع: الثمال والثرق والجنوب والثمال، ومن ثم حساب الاستهالكا السنوي عندما تكون

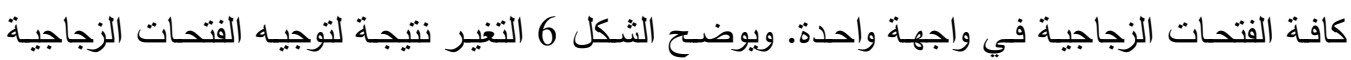

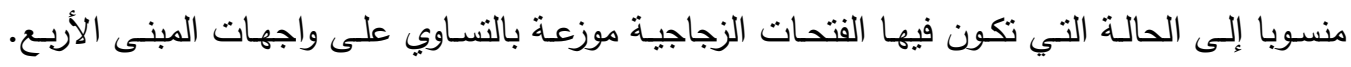

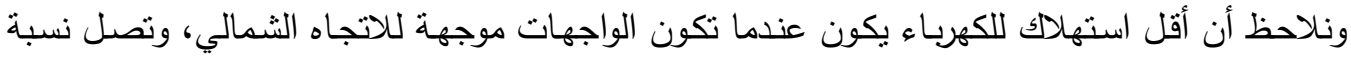
التغير إلى ما يقارب ال2.5\% لمبنى غير معزول حراريا. وهذه المقارنة تمثل حالة مثالية فعادة توجد

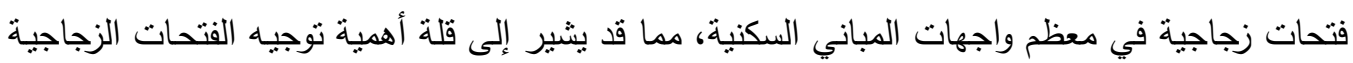

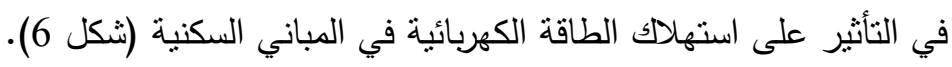

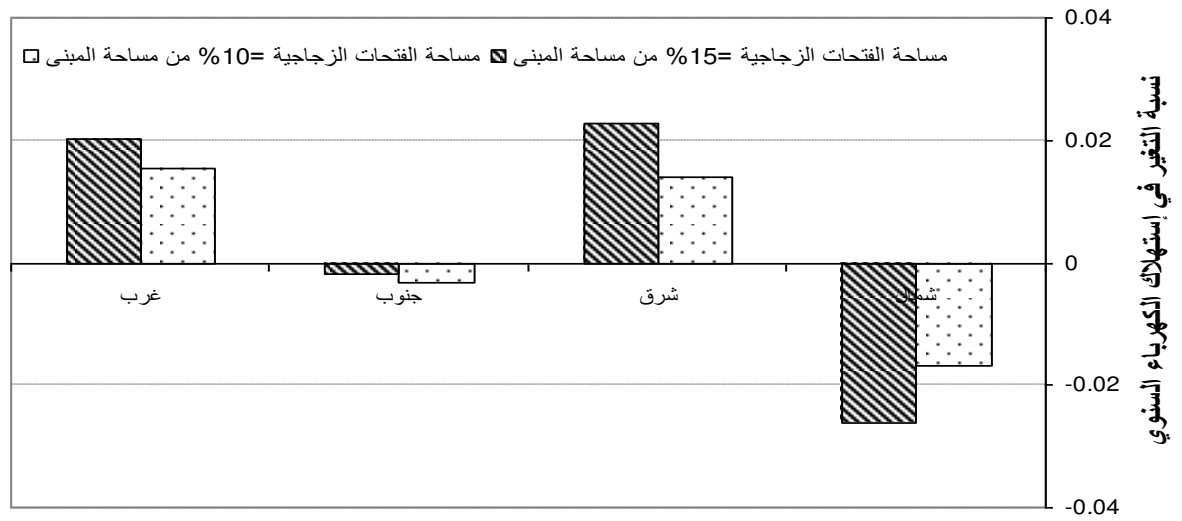

شكل 6 التغير في استهلاك الطاقة الكهربائية السنوية نتيجة لتغير توجيه الفتحات الزجاجية مقارنة بمبنى

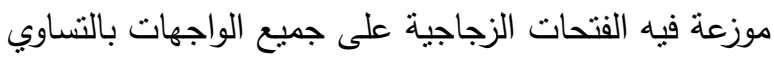




\section{3-3 كاسرات أثعة الثمس (shading devices)}

لفهم أثر استخدام كاسرات أثُعة الثمس المباشرة على استهلاك الطاقة الكهربائية نم تحليل أداء استهلاك الطاقة لمبنى سكني ذو فتحات زجاجية بمساحات تتراوح بين 10\% و 20\% من من مساحة المبنى. ويوضح الثكل 7 المتغيرات المستخدمة في تصميم كاسرات أثنعة الثمس. فالفتحات الزجاجية المستخدمة كانت

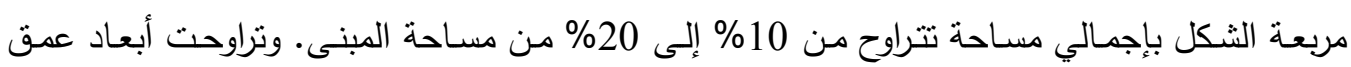
كاسرات من ثلث ارتفاع النافذة إلى كامل ارتفاع النافذة. ولقد أظهرت النتائج أهمية استخدام كاسرات

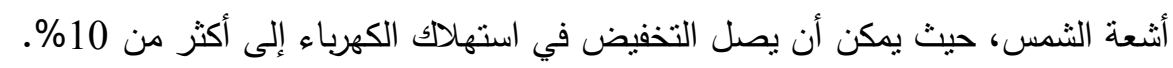

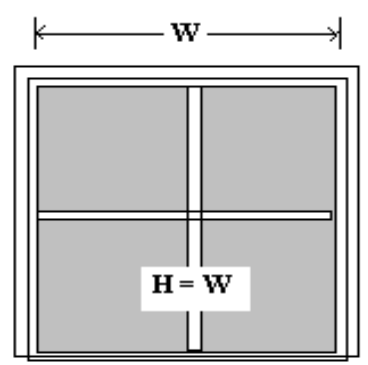

ELEVATION

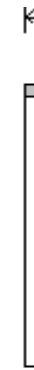

\section{SECTION}
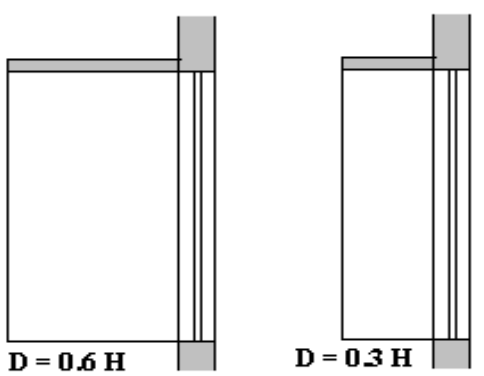

شكل 7 رسم توضيحي يبين متغيرات كاسرات أثنعة الثمس المستخدمة في الدراسة

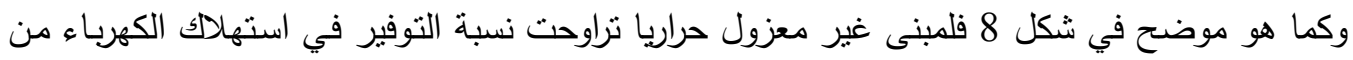
ما يقارب 3\% إلى 5\% لمبنى بمساحات زجاجية تساوي 10\% من من مساحة المبنى. وترتفع هذه النسبة

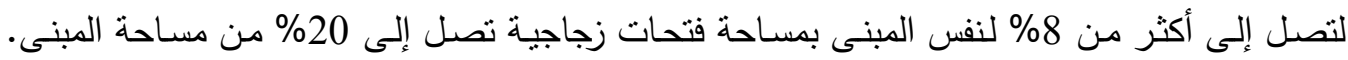

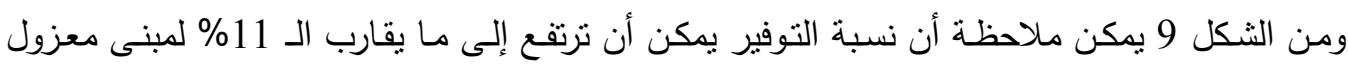
حراريا وبمساحة فتحات زجاجية تناوي 20\% من من مساحة المبنى.

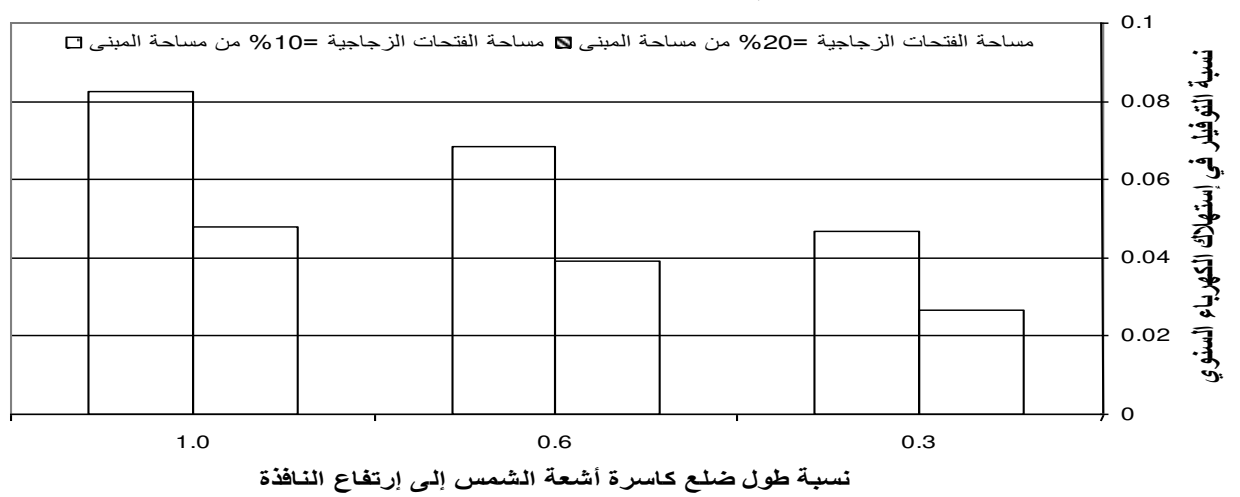

شكل 8 التوفير في استهالك الطاقة الكهربائية السنوية نتيجة لاستخدام كاسرات أثثعة الثنمس (مبنى غير الثراع معزول حراريا) 


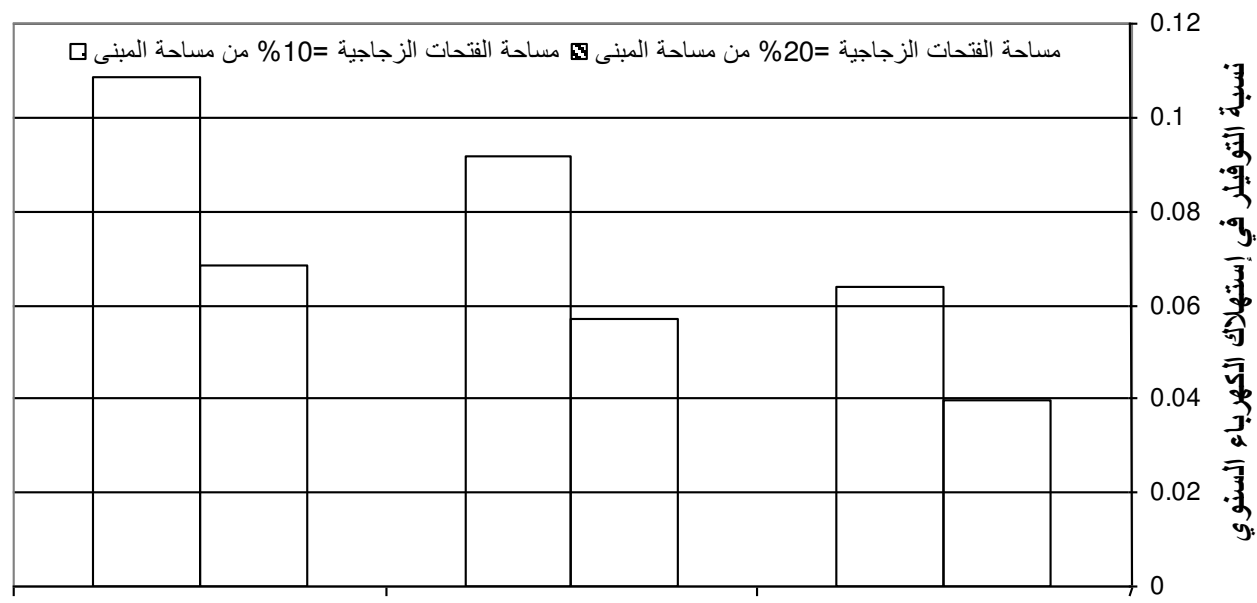

1.0

0.6

0.3

نسبة طول ضلع كاسرة أشعة الشمس إلى إرتفاع الناقذة

شكل 9 التوفير في استهلاك الطاقة الكهربائية السنوية نتيجة لاستخدام كاسرات أثنعة الثمس (مبنى الثنى

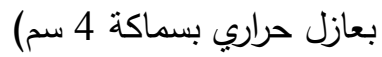

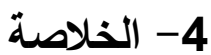

إن فهم تأثثير الخيارات المختلفة لتوفير استهلاك الطاقة الكهربائية في المباني السكنية هو أمر مهم للمهندس المعماري ومالك المبنى لاتخاذ قرارات تصميمية مبنية على أساس علمي. فاستخدام العازل الحراري ليس بالضرورة أن يكون هو الخيار الأهم. فمن خـلال هذه الدراسـة تم تحليل تأثنير الفتحات

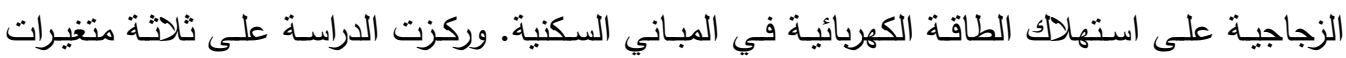
رئيسية شملت مساحة وتوجيه الفتحات الزجاجية إضافة إلى استخدام كاسرات أثنعة الثمس. وأظهرت

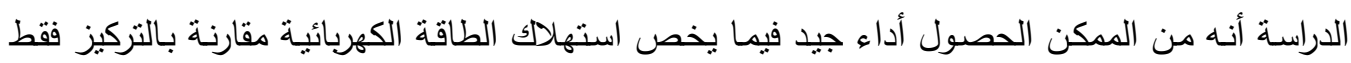

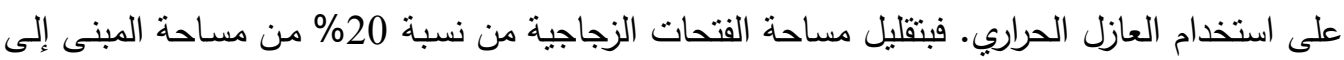
16\% يمكن الحصول على تخفيض في استهلاك الطاقة الكهربائية السنوية بما يعادل 4\% للحصول

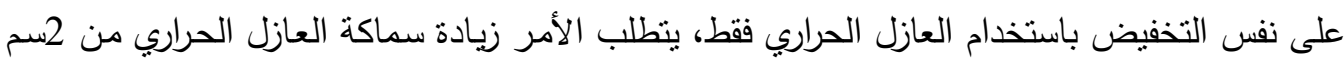

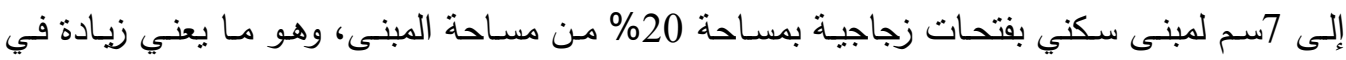

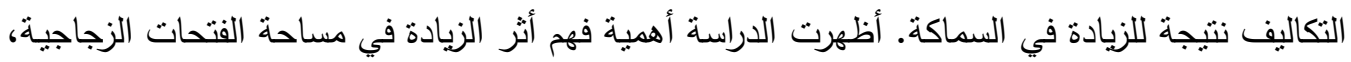

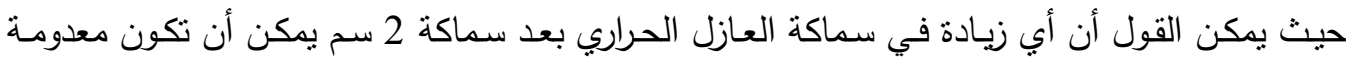

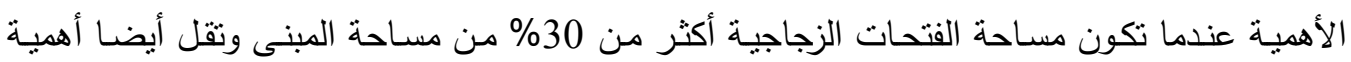

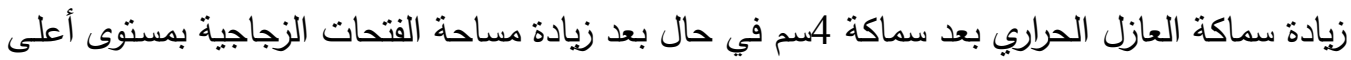
من 10\% من مساحة المبنى. 
وأظهرت النتائج الأثر المحدود فيما يخص توجيه الفتحات الزجاجية، ومـع هذا يظل خيار الواجهات الثمالية هي الأفضل دائما فيما يخص استهلاك الطاقة الكهربائية السنوية. كما أظهرت الدراسة الأثر الثراته

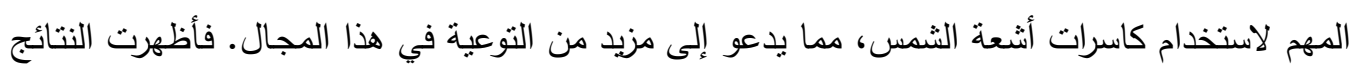

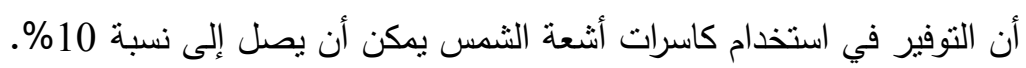

[1] Al-Ajalan, S., Al-Ibrahim, A., Adulkhaleq, M. and Alghamdi, F. (2006) "Developing sustainable energy policies for electrical energy conservation in Saudi Arabia", Energy Policy 34,1556-1565.

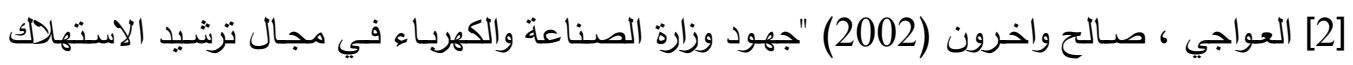

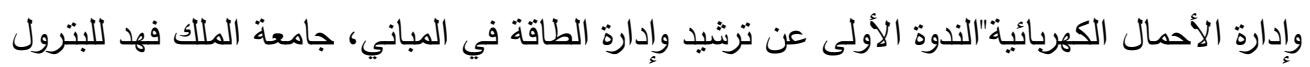

$$
\begin{aligned}
& \text { والمعادن ، الظهران، ذو القعدة. } \\
& \text { [3] الثركة السعودية للكهرباء. التقرير السنوي 2006. }
\end{aligned}
$$

[4] Numan, M. Y. F. Al-Maziad A. and Al-Khaja, W.: Architectural and Urban Design Potentials for Residential Building Energy Saving in The Gulf Region, Applied Energy, vol. 64, no.1-4, Sept-Dec. 1999, pp.401-410.

[5] Numan, M.; Al-Naim, M; Alshaibani, K. and Al-Maziad, F.:The Impact of Dynamic cultural Changes of The Design and Energy Performance of Residential Buildings in Saudi Arabia. Mediterranean Conference COMPLEX'2k, Energy and Environment. Beirut - Lebanon. 16 -18 Nov. 2000.

[6] Ahmad, A. and Elhadidy, M. Effect of residential buildings consumption on total power demand at SEC-ERB, The First Symposium on Energy Conservation \& Management in Buildings (31-42), King Fahd University of Petroleum and Minerals, Dhrhan, 2002.

[7] Almaziyad, F., Numan, M., Alshaibani, K. and Alnai, M. Windowless environment concept for saving of moseque's electrical consumption' The First Symposium on Energy Conservation \& Management in Buildings (31-42), King Fahd University of Petroleum and Mierals, Dhrhan, 2002.

[8] Al-mofeez, I 'Electrical energy consumption pre- and post- energy conservation measures: a case study' The First Symposium on Energy Conservation \& Management in Buildings (31-42), King Fahd University of Petroleum and Mierals, Dhrhan, 2002.

$$
\begin{aligned}
& \text { [9] الثيبياني، خالا ومحمد نعمان وإبراهيم المفيز وفارس المزيد، الفتحات الزجاجية والعزل الحراري في } \\
& \text { المملكة العربية السعودية: دراس مقارنة، بحث مقدم لمجلة جامعة الملك عبدالعزئ لعزيز }
\end{aligned}
$$

